\title{
Frontières
}

\section{Mort, ce lieu commun}

Comment en faire état?

\section{Daniel Castillo Durante}

Volume 18, numéro 2, printemps 2006

La mort dans tous ses états

URI : https://id.erudit.org/iderudit/1073213ar

DOI : https://doi.org/10.7202/1073213ar

Aller au sommaire du numéro

\section{Éditeur(s)}

Université du Québec à Montréal

ISSN

1180-3479 (imprimé)

1916-0976 (numérique)

Découvrir la revue

Citer cet article

Castillo Durante, D. (2006). Mort, ce lieu commun : comment en faire état ?

Frontières, 18(2), 7-9. https://doi.org/10.7202/1073213ar
Résumé de l'article

Notre société marchande contemporaine stéréotype le rapport à la mort. Incapable de projeter sa conscience au-delà d'une temporalité imposée par la consommation, le sujet actuel se refuse à penser sa propre mort. Afin d'échapper au temps et au lieu que la mortalité fixe comme frontière radicale, il sombre dans une économie de la copie que le caractère spectaculaire des sociétés occidentales ne fait qu'exacerber. Le présent article s'interroge sur ce phénomène.
Ce document est protégé par la loi sur le droit d'auteur. L'utilisation des services d’Érudit (y compris la reproduction) est assujettie à sa politique d'utilisation que vous pouvez consulter en ligne.

https://apropos.erudit.org/fr/usagers/politique-dutilisation/ 


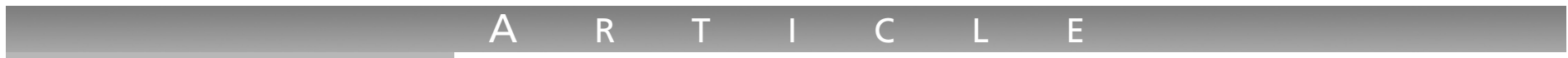 \\ MORT, \\ CE LIEU COMMUN COMMENT EN FAIRE ÉTAT?}

Résumé

Notre société marchande contemporaine stéréotype le rapport à la mort. Incapable de projeter sa conscience au-delà d'une temporalité imposée parla consommation, le sujet actuel se refuse à penser sa propre mort. Afin d'échapper au temps et au lieu que la mortalité fixe comme frontière radicale, il sombre dans une économie de la copie que le caractères pectaculaire des sociétés occidentales ne fait qu'exacerber. Le présent article s'interroge sur ce phénomène.

Mots clés: représentations - altérité dépouille.

\section{Abstract}

Our contemporary consumer culture structures a relationship with death. Unable to project one's consciousness beyond the imposed temporality of the consumer ethic, today's individual refuses to contemplate his or her own mortality. In an attempt to escape the radical frontier that death sets, today's individual is drawn into a logic of the copy, a logic spectacularly mastered by Western society. The present paper reflects on this situation.

Keywords: representations - alterity mortal remains.

\author{
Daniel Castillo Durante, Ph. D., \\ professeur, Département des lettres françaises, \\ Université d'Ottawa.
}

Le passage de vie à trépas, en Occident en tout cas, a souvent été stéréotypé sous la forme d'une crispation terrifiée de l'être en proie à l'agonie de sa propre conscience. Tout se passe comme si cette mort-là, la Parque, la Grande Faucheuse, voulait nous dérober notre bien le plus précieux. Elle est soustendue ainsi par une logique de soustraction qui voile paradoxalement ce qu'elle peut contenir de don ou, tout au moins, d'échange. Le souffle de la mort, la radicalité du geste qu'elle pose en nous enlevant la vie, met en place un mécanisme de transfert que l'Occident, souvent crispé et suspendu aux lèvres de la terreur, s'est jusqu'ici refusé à penser. Est-ce que cela veut dire que le phénomène de la mort doit aussi être pensé à partir de la mort? J'ai déjà dit ailleurs que jamais la vie ne m'a semblé aussi intense que lorsque je me rêvais mort (Castillo Durante, 2005). Se glisse ici la possibilité d'une expérience de la mort qui, en dépit de ses transgressions, opère le transfert à partir de la rive ontologique où la conscience gît. Peut-on aller plus loin afin de comprendre l'altérité de la mort? Quelle attitude de déprise et d'écoute à la fois serait-il envisageable d'adopter en vue d'une interaction qui tout en abolissant l'écoulement chronométré d'une conscience aux prises avec la perte parviendrait à une temporalité affranchie de frontières, ouverte à une altération sans cartographie possible? Peut-on donc penser la mort en fonction de ce qui en elle fait signe de vie? Pourquoi l'Occident s'acharne-t-il à ne la représenter que dans un rapport d'opposition avec la vie? Il n'est pas ici question de stéréotyper la mort en fonction de quelques étiquettes faisant partie du lexique positiviste médicolégal du genre «nécrophilie» et j'en passe. La mort comme transfert pourrait justement déplacer l'accent lourdement posé sur la perte pour faire ressortir la logique d'altération sans laquelle le rapport avec l'altérité reste scellé. C'est ici, si l'on peut dire, que le bât blesse. C'est ici aussi que l'on constate que, face à cette attitude de crispation ontologique, ce qui pose problème n'est peut-être pas la mort mais plutôt la vie. Le sens en tout cas que nous lui accordons. Et c'est là la première perspective herméneutique que l'on peut adopter à l'égard de ce lieu commun qu'est la mort.

\section{MORT ET ALTÉRITÉ}

La mort, en ce qui me concerne, ouvre sur une démarche réflexive qui ne peut être que recueillement, humilité, si j'ose dire, de premier communiant, ce seuil qu'on franchit à l'âge où la conscience s'éveille à peine à la perte mais ce seuil aussi, l'ultime communion, où le sujet sombre dans l'inconnu. Quel espace d'altérité la mort ouvre-t-elle ici ? Pour moi, le concept d'altérité, outre le rapport à autrui, à l'autre, au temps autre et à l'espace autre, renvoie aussi et surtout à l'altération, à cette dynamique qui transforme le sujet au point de le rendre méconnaissable à soi-même. Dans l'espace de la mort, la méconnaissance est de rigueur. Le familier n'y est que trompe-l'œil. S'efface ainsi le rapport au repère au profit d'une déprise de la vie qui, souvent brutalement, nous altère jusqu'à ce qu'il ne reste qu'une dépouille. La dépouille, statut paradoxal, signe ici le départ d'une conscience et l'avènement du détritus, la scorie, le pourrissable comme fruit d'une altération dont la mort est la logique vivante. Ce qui reste de nous, notre conscience ne peut que l'aborder dans une anticipation en relation asymétrique avec le phénomène. Altération tout d'abord de l'un par le radicalement Autre. Autre Anonyme aux prises avec la modification d'une conscience jusque-là souveraine. S'agit-il d'une limite au-delà de laquelle plus rien ne subsiste du conatus que Spinoza nous propose dans son Éthique (1999) comme le signe même du vivant? Ou bien 
avons-nous affaire à l'extinction pure et simple d'une conscience dont la dépouille n'interpelle nullement le silence des dieux? La mort en tant qu'extrême communion avec le néant est une image forte, ce qui, en dehors d'un petit côté tape-à-l'œil existentialiste, nous invite à considérer la mort comme lieu susceptible d'être abordé à partir d'une conscience encore active.

\section{MORT ET REPRÉSENTATION ARTISTIQUE}

Or l'éventualité d'une passerelle, aussi mince soit-elle, entre ce qui en moi fait signe de vie et l'au-delà a toujours fasciné la pratique artistique en général et littéraire en particulier. Je songe ici au roman Pedro Paramo du Mexicain Juan Rulfo ${ }^{1}$ dont l'enjeu principal est le dialogue entre les vivants et les morts. Tout s'y passe comme si, tant qu'on est en vie, le sujet ne parvenait pas à établir les passerelles capables de le sortir de l'isolement d'une conscience assiégée par la culpabilité et la frénésie d'une vie qui multiplie les pertes. Dans le contexte de la culture mexicaine, l'œuvre littéraire de Juan Rulfo matérialise probablement la tentative la plus réussie pour établir un lien entre l'approche catholique de la mort et celle, radicalement différente, des anciens Mexicains pour qui la mort, en règle générale, devait être pensée au sein de la communauté. Les sociétés contemporaines en se débarrassant des rituels et en marginalisant le rôle du religieux ont exacerbé encore un peu plus le divorce entre l'approche mexicaine de la mort et nos cérémonies funéraires sous-tendues essentiellement par des pratiques commerciales. En effet, la dépouille est confiée à des entreprises n'ayant aucun rapport avec le défunt et dont le mobile est purement et simplement le profit. Ce lien entre le profit et la mort, un tabou dont notre société évite de penser les conséquences (l'argent de la mort), en dit long sur le malaise et le malentendu qui existent entre le phénomène de la mort et les familles qui sont touchées. Dans la perspective mexicaine traditionnelle de la mort, la dépouille est prise en charge par l'ensemble de la communauté qui, tout en l'associant au cycle des récoltes, la célèbre comme un don qui doit retourner à la terre.

\section{LE DÉCLIN COMME FIGURE}

Dans la perspective matérialiste de nos sociétés de consommation, la vie est associée à l'expansion permanente de la croissance économique. Les sociétés dont les marchés sont perçus comme insuffisamment compétitifs ou en perte de vitesse sont analysées sous l'angle du déclin. Le déclin devient pour ainsi dire le symptôme avantcoureur d'une société vouée à sa perte. Subsumer tous les signes d'un prétendu déclin

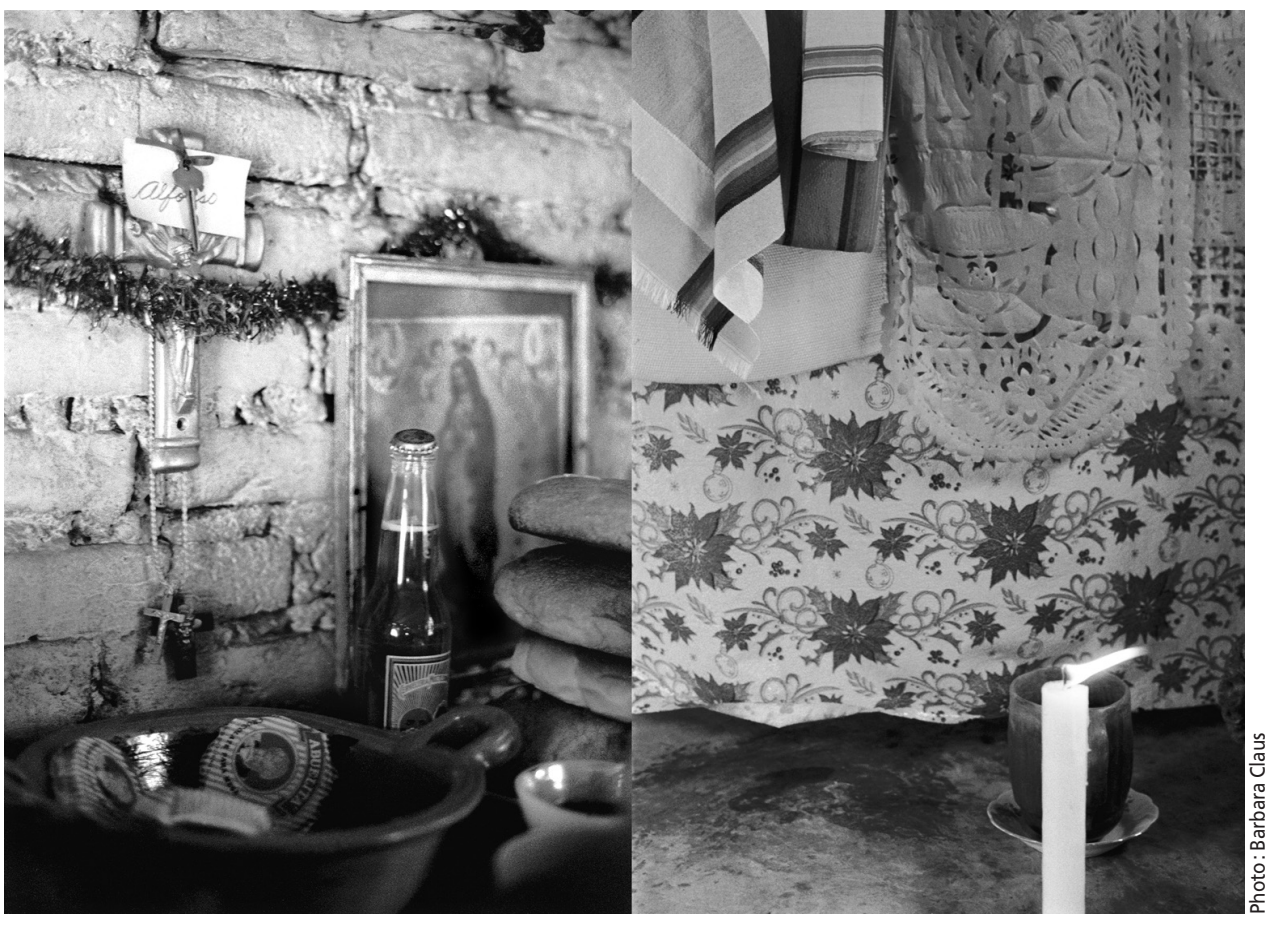

sous la forme de la fin est un stéréotype qui révèle en partie l'approche binaire et manichéenne du phénomène de la mort.

À force de nous tailler une place au soleil au nom de l'avoir - je suis ce que j'ai, ou, encore mieux: j'ai donc je suis (habeus ergo $s u m)^{2}-$, le vide existentiel et l'anémie du spirituel sont lus comme preuves irréfutables d'une chute inéluctable. Toujours dans le cadre de la représentation artistique, le cinéma québécois interroge cette imminence de la chute à partir du concept de déclin :

Les signes du déclin de l'empire sont partout. La population qui méprise ses propres institutions. La baisse du taux de natalité. Le refus des hommes de servir dans l'armée. La dette nationale devenue incontrôlable. La diminution constante des heures de travail. L'envahissement des fonctionnaires. La dégénérescence des élites...

Avec l'écroulement du rêve marxisteléniniste, on ne peut plus citer aucun modèle de société dont on pourrait dire: voilà comment nous aimerions vivre. Comme sur le plan privé, à moins d'être un mystique ou un saint, il est presque impossible de modeler sa vie sur aucun exemple autour de nous. Ce que nous vivons, c'est un processus général d'effritement de toute l'existence (Arcand, 1986, p. 143).

Denys Arcand, le cinéaste québécois, associe la mort en Occident à une exacerbation de l'individualisme que notre voisinage écrasant avec le néolibéralisme états-unien ne fait que renforcer. Dix-huit ans après son film Le Déclin de l'empire américain $^{3}$, le réalisateur reprend la suite à partir de la métaphore du "barbare ", dernier avatar récalcitrant d'un individua- lisme que la mondialisation des marchés projette comme signe universel de l'agonie $\mathrm{du}$ spirituel et de tous les rituels qui lui étaient rattachés. Les Invasions barbares ${ }^{4}$ pose ainsi le problème de la mort en fonction d'une approche désabusée du rapport à autrui miné par l'éclosion des valeurs qui menacent la solidarité dans le cadre d'une société vieillissante en proie à une crise majeure de ses institutions. Il est intéressant de noter que l'œil de la caméra en pétrifiant chaque photogramme fixe avant la lettre l'image dans une temporalité qui singe celle de la mort. Serait-ce le paradoxe du cinéma que de donner l'impression de vie là où il n'y a qu'un déroulement d'images en arrêt de mort? Si j'évoque la parole cinématographique de Denys Arcand, c'est parce qu'il m'apparaît comme le créateur qui incarne peut-être le mieux le déchirement d'une société qui s'est affranchie des rituels religieux catholiques sans pour autant parvenir à un accès à la modernité américaine avec les ressources inhérentes à une puissance mondiale

Nous vivons dans une petite société en marge de l'empire américain. Ne nous faisons pas croire que tout d'un coup cela va devenir une belle société. Ce ne sera jamais vrai. Nous sommes tributaires de nos origines, de l'héritage de ces 60000 paysans français qui ont été coupés de la mère patrie, et de notre situation politique [...] (Coulombe, 1993, p. 120).

Cette conscience sociale liée à une certaine marginalité de la société québécoise explique probablement en partie le regard lucide et acerbe en même temps du cinéaste dont les œuvres témoignent d'une préoccupation croissante à l'égard de la mort. 


\section{NOM, ANONYME, MARGINAL}

On parle de la pulsion de mort, du désir de mort, mais quel est le désir de la mort, quelle est cette logique dont les représentations sont incapables d'en saisir les fondements? La multiplicité des masques qui voilent notre rapport à la mort fait état d'une dialectique qui échappe à la reconnaissance. Alors que dans son interaction avec le même, l'autre cherche à faire reconnaître son désir, la mort, quant à elle, ne peut être coiffée que d'étiquettes médicales: mort d'un cancer, foudroyé d'un infarctus du myocarde, achevé par une cirrhose alcoolique. L'idée ne viendrait à personne de dire: décédé d'une rencontre longuement recherchée avec le désir de la mort. Lorsqu'on est en santé et bien portant, le lien à la mort nous est présenté comme absurde tandis que c'est probablement à ce moment-là que le rapport de force, si l'on peut dire, est le meilleur pour une saisie du phénomène. Une approche de la mort qui ne passe pas par le stéréotype, présuppose de s'engager dans un dialogue avec l'anonyme. En ce sens, l'anonyme peut être considéré comme la part cachée de l'altérité. Le mot vient du latin anonymus. Il s'agit d'un emprunt du grec nomos qui veut dire nom et loi tout à la fois. Le «a » privatif pourrait donc renvoyer autant à l'absence de patronyme qu'à un statut de hors-jeu. L'anonyme se soustrait ainsi au dédale de filiations tout en se mettant à l'abri de la loi. Dans cette perspective, l'altérité gagnerait à être thématisée comme une cartographie possible de stratégies permettant de se dérober aux représentations du même. Cette possibilité offerte à l'anonyme de ne pas jouer le jeu comme tout le monde expliquerait-elle d'une certaine manière la violence qui cherche à effacer chez l'autre ce que l'on ne peut pas saisir? Dans ce contexte, l'anonyme serait cet aspect de l'altérité capable de déjouer les pièges d'une dialectique de reconnaissance qui exige une conformité tacite avec les intérêts du système de représentation en place.

\section{LA MORT: L'AUTRE ANONYME}

En postulant que la mort peut être avant tout l'altération de l'un par l'Autre Anonyme, je m'interroge sur ce passage du connu, le tangible et nommable - le patronyme et le patrimoine - vers l'anonyme, le dépotoir de la reconnaissance. Pure sommation muette, la mort évite le nom car, dans sa force aveugle de transfert, elle ne reconnaît personne. En révoquant la souveraineté de notre conscience individuelle, elle nous assujettit au lieu commun mais libère en même temps ces restes à partir desquels il nous faudra dorénavant interpréter le monde. Interpréter le monde à partir de la dépouille $\mathrm{du}$ père est au demeurant le geste fondateur de la loi. C'est probablement ce rapport de la mort à la loi qui fonde, d'un point de vue phénoménologique, le stéréotype (stereos, pater $^{5}$ ), c'est-à-dire le stereos, le solide, le dur, du typus ("modèle», «symbole»), ou le tupos ("empreinte», «marque»). En ce sens, le stéréotype, tout comme la mort, est un geste qui se répète ; dans le silence glacé des saisons, il révèle le nom comme lieu de représentation truquée, demeure du factice où le sujet affirme une identité qui le rattache à un ordre symbolique que la mort gomme. Les cimetières avec leurs épitaphes gravées dans la pierre sont là pour que le visiteur, rare et parfois pressé, fasse l'appel de mémoire onomastique que lui dicte une conscience souvent coupable. Cet abattoir de noms ayant mordu la poussière illustre à certains égards l'impasse d'une société qui a du mal à penser la mort autrement que par des mécanismes, c'est le cas de le dire, de pétrification. La fosse commune s'érige ici comme le dernier outrage à la mémoire du défunt. Rien ne désespère davantage l'homme occidental que l'anonymat de la

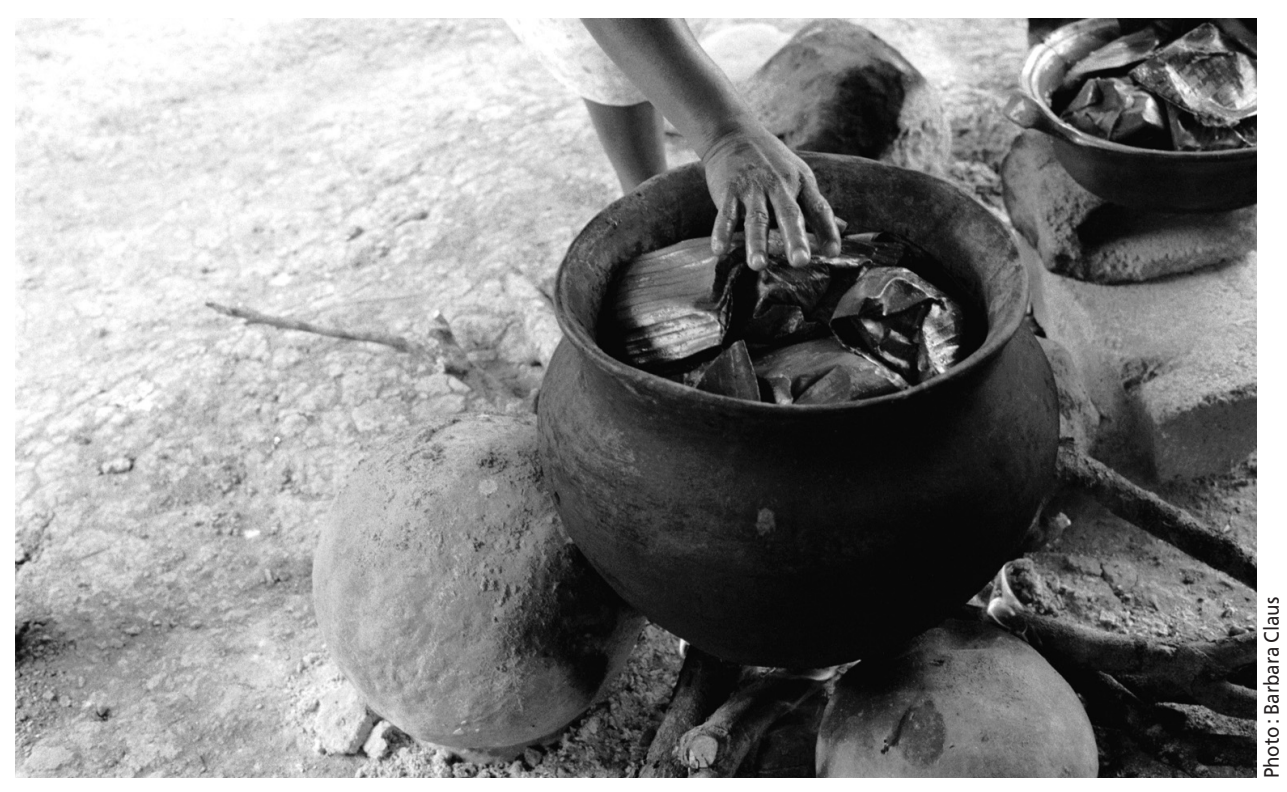

mort, car elle nargue son besoin pérenne de clicher tout ce qui lui échappe. En ce sens, la mort se présente comme marginale, en bordure de la société du spectacle (Debord, 1987, 1988). Elle opère dans un registre aléatoire qui ignore le principe d'assurance qu'exige la société occidentale pour continuer à «rouler». L'anonyme de la mort installe le précaire, le stochastique et le marginal comme alternatives proprement finales face à une programmation qui, paradoxalement, finit par faire de la vie un réseau d'accès à des reconnaissances de plus en plus fragmentaires. Les mots de passe devenant proliférants, le seul password qui se dérobe à notre emprise technologique est précisément celui de la mort dont l'anonymat nous nargue et nous assujettit à une attente anxieuse et souvent crispée. Hostile à une véritable mise à l'épreuve de l'Anonyme autre, la société occidentale en est à se souhaiter une mort rapide et indolore. La dimension épistémologique, la sommation de son altération, y sont donc passées sous silence. Or si pensée de la mort il y a, elle ne peut se faire que dans un espace de détachement qui exige une révocation en doute des représentations généralement en noir et blanc de ce phénomène extrême.

\section{BIBLIOGRAPHIE}

ARCAND, D. (1986). Le Déclin de l'empire américain, Montréal, Boréal.

COUlOMBE, M. (1993). Denys Arcand. La vraie nature du cinéaste, Montréal, Boréal.

DEBORD, G. (1987). La société du spectacle, Paris, Éditions Lebovici.

DEBORD, G. (1988). Commentaires sur la société du spectacle, Gallimard, Paris.

CASTILLO DURANTE, D. (2005). "Célébrer la mort? Mélancolie et représentation », Frontières, Volume 18, numéro 1, automne 2005, p. 6.

CASTILLO DURANTE, D., (1994). Du stéréotype à la littérature, $\mathrm{XYZ}$, Montréal.

SPINOZA, B. (1999), Éthique, Paris, Seuil.

\section{Notes}

1. Juan Rulfo. Écrivain mexicain (Sayula, 1918). Auteur du recueil de nouvelles El Llano en llamas (Le Llano en flammes, 1953) et de Pedro Paramo (1956), l'un des romans les plus importants de la littérature latino-américaine $\mathrm{du} \mathrm{XX}^{\mathrm{e}}$ siècle.

2. Ici, la formule de René Descartes (Cogito ergo sum) se dévoile transformée par une société dont les critères de rentabilité immédiate surdéterminent l'interpellation de l'individu en sujet productif.

3. Le film Le Déclin de l'empire américain, réalisé en 1986 par Denys Arcand, est le film québécois ayant eu le plus grand rayonnement international.

4. Le film Les Invasions barbares, réalisé par Denys Arcand et produit par Cinémaginaire, est sorti en salles le 9 mai 2003.

5. Castillo Durante, 1994. 